\title{
Magic vaginal douche for severe vaginal mucositis: a case-based approach
}

\author{
Queenjal Anandi, Shrenik Ostwal, Himanshu Patel, Priti Sanghavi \\ Department of Palliative Medicine, Gujarat Cancer and Research Institute, Ahmadabad, India
}

Correspondence: Dr. Shrenik Ostwal,Assistant Professor, Department of Palliative Medicine, Gujarat Cancer and Research Institute, Ahmadabad-380016, Email drshrenikostwal@gmail.com

Received: May 16,2018 | Published: June II, 2018

Copyright@ 2018 Anandi et al. This is an open access article distributed under the terms of the Creative Commons Attribution License, which permits unrestricted use, distribution, and reproduction in any medium, provided the original author and source are credited.

\begin{abstract}
Vaginal mucositis is commonly seen in patients with recto-vaginal fistula, significantly affecting their quality of life. A 45 -year-old woman diagnosed with a case of metastatic cervical cancer developed severe vaginal mucositis due to recto-vaginal fistula. In spite of repeated counselling for diversion colostomy as a definitive treatment, the patient denied the active intervention. Standard measures to control vaginal irritation, including analgesic dose escalation, were found to be ineffective in her case. So, we modified vaginal douche with addition of lignocaine viscous and two crushed tablets of morphine (Magic Vaginal Douche) for better symptom control. This combination was found to have significant benefit in controlling vaginal mucositis, reducing opioid dose escalation, and thus improving her quality of life.Patients with vaginal mucositis whose symptoms are not controlled with standard measures can be treated with this combination for better symptom profile and quality of life.
\end{abstract}

Keywords: Vaginal douche, Vaginal mucositis, Vaginitis

\section{Introduction}

Vaginal mucositis, also known as vaginitis, is a common problem in patients with gynaecological cancers, more commonly seen in recto vaginal fistula. ${ }^{1}$ Pathophysiology includes irritation of vaginal mucosa due to chemotherapy, radiotherapy, local infection and irritating

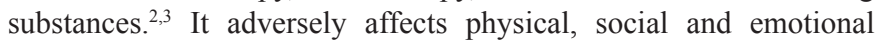
functioning. Common measures used to control vaginal irritations is removal of precipitating cause, maintaining local hygiene, control of infections and use of adequate analgesia. We report a case of a 45-year-old woman suffering from severe vaginal mucositis due to recto vaginal fistula, and denying to undergo any active intervention.

\section{Case summary}

A 45-year-old widowed lady, living with her unmarried son, was diagnosed with squamous cell carcinoma of cervix. She had received chemotherapy and radiotherapy as disease modifying treatment. After a disease-free interval of 6 months, she had recurrence with metastasis to liver and peritoneum. She received palliative chemotherapy for the same. Following this, she developed deep vein thrombosis of lower limb for which symptomatic treatment was started. After being asymptomatic for few days, she presented to emergency department with history of rectal bleeding which subsided following hydrocortisone enema and supportive measures. She was referred to the palliative medicine department for symptomatic treatment. During her first visit to palliative care, she complained of having severe pain at hypogastric area (NRS- 8-9/10), non-radiating, mixed type with both nociceptive and neuropathic component, causing disturbance in her sleep and activities of daily living. She was started on oral Morphine
$10 \mathrm{mg}$ every 4 hours with $10 \mathrm{mg}$ as extra dose for breakthrough pain. Other supportive medications- paracetamol, pregabalin, laxatives and antacids- were also prescribed. With inadequate pain relief even after 48 hours of oral medications, her opioid dose was increased to 15 mg every 4 hours. Her symptoms were well under controlled for next one week. After one week, her pain increased in intensity; hence a decision for superior hypogastric block was advised. With normal laboratory parameters, she underwent a nerve block. The procedure was uneventful without any adverse events. Post-procedure her opioid requirement decreased significantly to $5 \mathrm{mg}$ Q4hrly. After 2 weeks of a stable oral dose, she re-developed pain at the same site. Her opioid dose was escalated giving good symptom control.

A few days later she presented to outpatient department with rectovaginal fistula with severe burning pain (NRS- 10/10) at vaginal area. It was not associated with any vaginal bleeding. On examination, she was afebrile with pulse rate of $124 / \mathrm{min}$ and respiratory rate of $22 / \mathrm{min}$. A vaginal examination showed feculent matter with sero-sanguinous discharge, severe erythema of vaginal mucosa and edematousvulval area. Her pain settled with $6 \mathrm{mg}$ of intravenous morphine. She was counseled in details and was advised for diversion colostomy. She refused any active intervention or procedures. The patient and caregiver were advised and educated for vaginal douche and sitz bath (povidone iodine followed by $\mathrm{KMnO}_{4}$ ). The opioid dose was escalated to provide adequate analgesia (Total daily dose $=120 \mathrm{mg} /$ day). In spite of repeated counselling, she refused any hospital or hospice care. With caregiver's repeated hospital visits in view of her inadequate pain control, her opioid dose and other supportive medications were escalated frequently to optimize analgesia (Total oral morphine required $=180 \mathrm{mg} /$ day). But, she denied any significant pain relief in 
her mucositis. This has led to significant distress to patient and her son. Her son left his job to care for his mother, so a home care plan was given.

At home she received mixture of injectable Metronidazole, povidone iodine, lidocaine viscous and two crushed tablets of $10 \mathrm{mg}$ morphine vaginally. Her pain reduced significantly (NRS-1/10) within the next 10-15 min. She was advised to continue Magic Vaginal Douche three times daily. We observed significant relief in her symptoms with very minimal need for oral dose escalation. Her opioid requirement decreased to $120 \mathrm{mg} /$ day. With multiple and repeated counselling, she agreed to a diversion colostomy and is scheduled for the same. Currently she is on morphine $120 \mathrm{mg}$ /day in divided doses, paracetamol $3 \mathrm{mg} /$ day, pregabalin $300 \mathrm{mg} /$ day and other supportive measures with her symptoms well under control.

\section{Discussion}

Mucositis is the inflammatory reaction that occurs when cancer treatments break down the continually dividing epithelial cell linings, leaving the mucosal tissue open to ulceration and infection. Common risk factors include cancers involving areas of mucous membranes as a complication of chemotherapy - radiotherapy, and vaginal irritation by substances such as faecal matter, urine, infections or foreign bodies. ${ }^{4}$ Additional risk factors for vaginal mucositis include: old age, poor self-care dehydration, and poor nutritional status. Consequently, it can lead to vaginal symptoms like bleeding, pain, diarrhoea, itching, discharge and other discomfort. This can adversely affect a woman's physical, psychosocial, emotional and sexual functioning. Regular and frequent assessment are important in preventing complications and thus improving quality of life.

Sitz bath with povidone iodine and vaginal douche are commonly advised practices in the palliative care setting for local hygiene. .,2, $^{-2}$ Vaginal douching consists of intravaginal cleansing with liquid solutions. Povidone iodine acts as antiseptic ${ }^{7,8}$, while metronidazole acts on anaerobic flora leading to its antibacterial action. This prevents foul smells and discharge.

In this case, our patient had clinical mucous membrane irritation and an inflammatory reaction. Standard measures had been attempted for this patient. With inadequate benefit, we tried the addition of lignocaine viscous and crushed morphine in the solution. Lignocaine and morphine are commonly used as local measures for oral mucositis. Various studies have proven their benefit in controlling oral mucositis ${ }^{9-14}$. Lignocaine acts via inhibiting $\mathrm{Na}+$ channels, hence exerting its anaesthetic action. Morphine, being a strong opioid, exerts its analgesic action via acting on $\mu$ receptors. Based on same principles for oral mucositis, we found her pain relieved significantly and she did not require any opioid dose escalation. It reduced her pill load and therefore prevented further side effects of medications. With adequate relief in her symptoms, she becomes physically functional. This reduced her dependency on family members and increased her quality of life significantly. Also, her son was able to resume his daily routine.

Increased awareness, education, and symptom management of negative vaginal side effects (vaginal irritation and mucositis) are important parts of patient care, which may ultimately improve overall functioning and quality of life. To our best knowledge, this is the first case report using combination of metronidazole, povidone iodine, lignocaine viscous and morphine (i.e. Magic Vaginal Douche) for controlling symptoms from severe vaginitis. However, more robust and prospective studies are required for establishing its benefit in palliative care settings. We recommend use of magic vaginal douche as an alternative in patients who deny any definite intervention for their symptoms.

\section{Conflicts of interest}

There are no conflicts of interest for this study.

\section{Ethical approval}

Not required.

\section{References}

1. Friedman JH. Chief complaint. Med Health R I. 2011;94(2):1188.

2. Krychman ML, Carter J, Aghajanian CA, Dizon DS, Castiel M. Chemotherapy-induced dyspareunia: A case study of vaginal mucositis and pegylated liposomal doxorubicin injection in advanced stage ovarian carcinoma. Gynecol Oncol. 2004;93(2):561-563.

3. Zelga P, Tchórzewski M, Zelga M, Sobotkowski J, Dziki A. Radiationinduced rectovaginal fistulas in locally advanced gynaecological malignancies-new patients, old problem? Langenbeck's Arch Surg. 2017;402(7):1079-1088.

4. Herdman C, Levin K, Dzuba I, Castro W, Muhombe K, Sellors J. Palliative Care for Women With Cervical Cancer: A Field Manual. PATH (A Catal Glob Heal. 2003:1-89.

5. Manuscript A, Dysfunction E. NIH Public Access. 2015;25(8):713-724.

6. Chu TY, Chang YC, Ding DC. Cervicovaginal secretions protect from human papillomavirus infection: Effects of vaginal douching. Taiwan $J$ Obstet Gynecol. 2013;52(2):241-245.

7. https://www.ncbi.nlm.nih.gov/pubmed/1495688

8. Pavlova SI, Tao L. In vitro inhibition of commercial douche products against vaginal microflora. Infect Dis Obstet Gynecol. 2000;8(2):99-104.

9. McGuire DB, Fulton JS, Park J, et al. Systematic review of basic oral care for the management of oral mucositis in cancer patients. Support Care Cancer. 2013;21(11):3165-3177.

10. Saunders DP, Epstein JB, Elad S, et al. Systematic review of antimicrobials, mucosal coating agents, anesthetics, and analgesics for the management of oral mucositis in cancer patients. Support Care Cancer. 2013;21(11):3191-3207.

11. Chan A, Ignoffo RJ. Survey of topical oral solutions for the treatment of chemo-induced oral mucositis. J Oncol Pharm Pract. 2005;11(4):139143.

12. CerchiettiLCA, NaviganteAH,BonomiMR, etal.Effect of topical morphine for mucositis-associated pain following concomitant chemoradiotherapy for head and neck carcinoma. Cancer. 2002;95(10):2230-2236.

13. Bhatt V, Vendrell N, Nau K, Crumb D, Roy V. Implementation of a standardized protocol for prevention and management of oral mucositis in patients undergoing hematopoietic cell transplantation. J Oncol Pharm Pract. 2010;16(3):195-204.

14. Dodd MJ, Dibble SL, Miaskowski C, et al. Randomized clinical trial of the effectiveness of 3 commonly used mouthwashes to treat chemotherapyinduced mucositis. Oral Surgery, Oral Med Oral Pathol Oral Radiol Endodontology. 2000;90(1):39-47. 